Article

\title{
A Cyclic Macro-Element Framework for Consolidation-Dependent Three-Dimensional Capacity of Plate Anchors
}

\author{
Anderson Peccin da Silva ${ }^{1, *}$, Andrea Diambra ${ }^{1}$ (D), Dimitris Karamitros ${ }^{1}$ and Shiao Huey Chow ${ }^{2}$ (D) \\ 1 Queen's School of Engineering, University of Bristol, Bristol BS8 1TR, UK; \\ andrea.diambra@bristol.ac.uk (A.D.); d.karamitros@bristol.ac.uk (D.K.) \\ 2 Department of Infrastructure Engineering, University of Melbourne, 01, 1.05, Engineering Block B, \\ Parkville, VIC 3010, Australia; shiaohuey.chow@unimelb.edu.au \\ * Correspondence: a.peccindasilva@bristol.ac.uk
}

Citation: Peccin da Silva, A.;

Diambra, A.; Karamitros, D.;

Chow, S.H. A Cyclic Macro-Element

Framework for

Consolidation-Dependent

Three-Dimensional Capacity of Plate

Anchors. J. Mar. Sci. Eng. 2021, 9, 199.

https://doi.org/10.3390/

jmse9020199

Academic Editor: Deborah Greaves

Received: 30 December 2020

Accepted: 10 February 2021

Published: 13 February 2021

Publisher's Note: MDPI stays neutral with regard to jurisdictional claims in published maps and institutional affiliations.

Copyright: (c) 2021 by the authors. Licensee MDPI, Basel, Switzerland. This article is an open access article distributed under the terms and conditions of the Creative Commons Attribution (CC BY) license (https:/ / creativecommons.org/licenses/by/ $4.0 /)$.

\begin{abstract}
This paper presents a new macro-element modelling framework for plate anchors which enables the effect of pore water pressure changes and the related evolution of soil strength during the process of cyclic loading and consolidation to be captured. The proposed modelling framework combines an advanced macro-element model for plate anchors, expanded to capture the cyclic loading behaviour, with a simple one-dimensional model of undrained shearing and consolidation for a soil element representative of the whole soil mass around the anchor. The representative soil element tracks the effects of changes in effective stress on the soil strength, which in turn governs the anchor capacity in the macro-element model. The two modelling components are linked through a mobilised capacity compatibility condition. It will be firstly shown that such modelling framework is able to capture the expected changes in an anchor's capacity related to cyclic pore pressure generation and consolidation under one-dimensional cyclic loading of the anchor. Then, the model will be used to explore the plate anchor's behaviour and failure mechanisms under loading conditions which mobilise its full three-dimensional cyclic loading capacity. The macro-element model will identify some conflicting mechanisms (i.e., the anchor's kinematic/rotation and soil weakening/strengthening) governing the three-dimensional capacity of the anchor.
\end{abstract}

Keywords: plate anchors; floating offshore structures; cyclic loading; soil consolidation; macroelement modelling; anchor kinematics

\section{Introduction}

Embedded anchoring systems are commonly used in offshore engineering to maintain the position of and provide stability to floating structures. While they have been largely used in the oil and gas industry for exploration in deep water sites, further interest has been recently sparked by the emergence of floating renewable energy devices. These include floating wind turbine developments to harness additional and stronger wind resources in deeper water sites as well as floating wave energy converters [1,2]. Among several anchoring options, vertical plate anchors are an attractive solution due to the provision of considerable holding capacity through their large embedded plate [3-5].

While the experimental study of a plate anchor's behaviour is typically carried out using centrifuge testing, e.g, [6,7], its modelling can be performed using numerical methods such as finite element analyses, e.g., [3,8]. However, numerical modelling of anchor keying and further loading is a challenging and time-demanding three-dimensional problem, which requires the careful handling of large mesh deformations associated with the anchor's displacements and of complex solutions for modelling the plate and soil interaction. The macro-element modelling technique is an alternative and time-effective method for obtaining an estimation of the geotechnical response and when seeking insight into some 
aspects of the geotechnical behaviour [9]. Macro-element models can also be implemented into and facilitate the integrated load analysis of offshore structures, as demonstrated by the macro-element REDWIN foundation models, i.e., [10,11].

Macro-element models have been developed for several geotechnical problems including shallow foundations, piles, retaining walls and anchors, among others [12-14]. However, their development typically considers either drained or undrained conditions, and the effects of the pore water pressure generation and consolidation processes, which affect the effective stress state and thus the soil strength, are not currently considered in available macro-element models. The consideration of these processes is particularly important in offshore geotechnical applications, when considering operational conditions and the whole design life of geotechnical systems [15-17]. Few macro-element models have included the hydro-mechanical effects to account for partial drainage, yet all in the context of penetrometers and shallow foundations [18,19]. Houlsby and Cassidy [18] used a mechanical analogy in which springs and sliders connected in series represent the elastic and plastic undrained deformations of the foundation, respectively. An additional spring represents the drained penetration, and it is connected in parallel to a dashpot that represents the drainage processes. The results from the model were compared to experimental data and it was shown to capture the effect of different load rates applied to a spudcan. Similarly, Flessati et al. [19] used a spring and a slider to represent the elastic and plastic deformations of a strip shallow foundation under undrained conditions. The partial drainage process was represented by a viscous damper (representing the drainage process) in parallel with a spring in series with a slider (to represent the elastic and plastic deformations during perfectly drained conditions).

In the context of anchoring systems, Chow et al. [7] demonstrated that the loading capacity of vertical plate anchors in sand is dependent on the rate of loading owing to the consolidation taking place during the loading process. The weakening of cohesive soils' strength during cyclic loading is also related to pore water pressure generation [20]. Yu et al. [21] and Singh and Ramaswamy [22] found that the vertical post-cyclic capacity of horizontal anchors in clay was lower than the monotonic capacity, but the capacity reduction was less pronounced for lower loading frequencies. Ponniah and Finlay [23] demonstrated that long periods of unloading after long-term cycles increased the anchor capacity during recycling. More recently, Zhou et al. [24] considerably expanded the knowledge on the evolution of soil strength during cyclic loading, proposing a new analytical framework to capture the effects of changes in the undrained shear strength of clays during cyclic loading with T-bar penetrometer tests and a spudcan footing installation. The framework, based on critical state concepts, was further applied to vertical anchors loaded horizontally and subjected to cyclic and maintained loads combined with consolidation [25]. This study demonstrated that the anchor capacity increases significantly when full consolidation takes place during maintained loading or during prolonged cyclic loading.

This paper presents a new strategy to account for changes in pore water pressures and effective stresses in the soil when employing a macro-element modelling approach. The modelling framework expands a previously developed macro-element model for the plate anchors [26,27] and combines it with a simple one-dimensional model of shearing and consolidation for a representative soil element around the anchor. The representative soil element tracks the effects of pore pressure generation and changes in effective stress on the soil strength, which in turn governs the anchor capacity in the macro-element model. While it will be also shown that the modelling framework is able to capture the expected changes in strength related to cyclic pore pressure generation and consolidation under one-dimensional anchor loading [25], the attention of this paper will be directed to a loading condition which triggers the full three-dimensional cyclic loading capacity of the anchor. Three-dimensional loading capacity is typically triggered in offshore plate anchors [3], and the macro-element model will be used to seek insight into the governing behaviour and mechanisms. While, due to the unavailability of experimental data in clay, the model predictions will not be quantitatively verified, it will be shown that the 
macro-element model will identify some fundamental and counteracting mechanisms (i.e., kinematic and rotation of the anchor, soil weakening or strengthening) governing the threedimensional bearing capacity of the anchor. These findings may provide inspiration for future experimental investigations and give an indication of some aspects and mechanisms to be considered in the three-dimensional cyclic assessments of plate anchors.

\section{Modelling Framework}

\subsection{Geometry and Definitions}

The assumed geometry of a generic plate anchor and the sign convention for forces and displacements are presented in Figure 1.

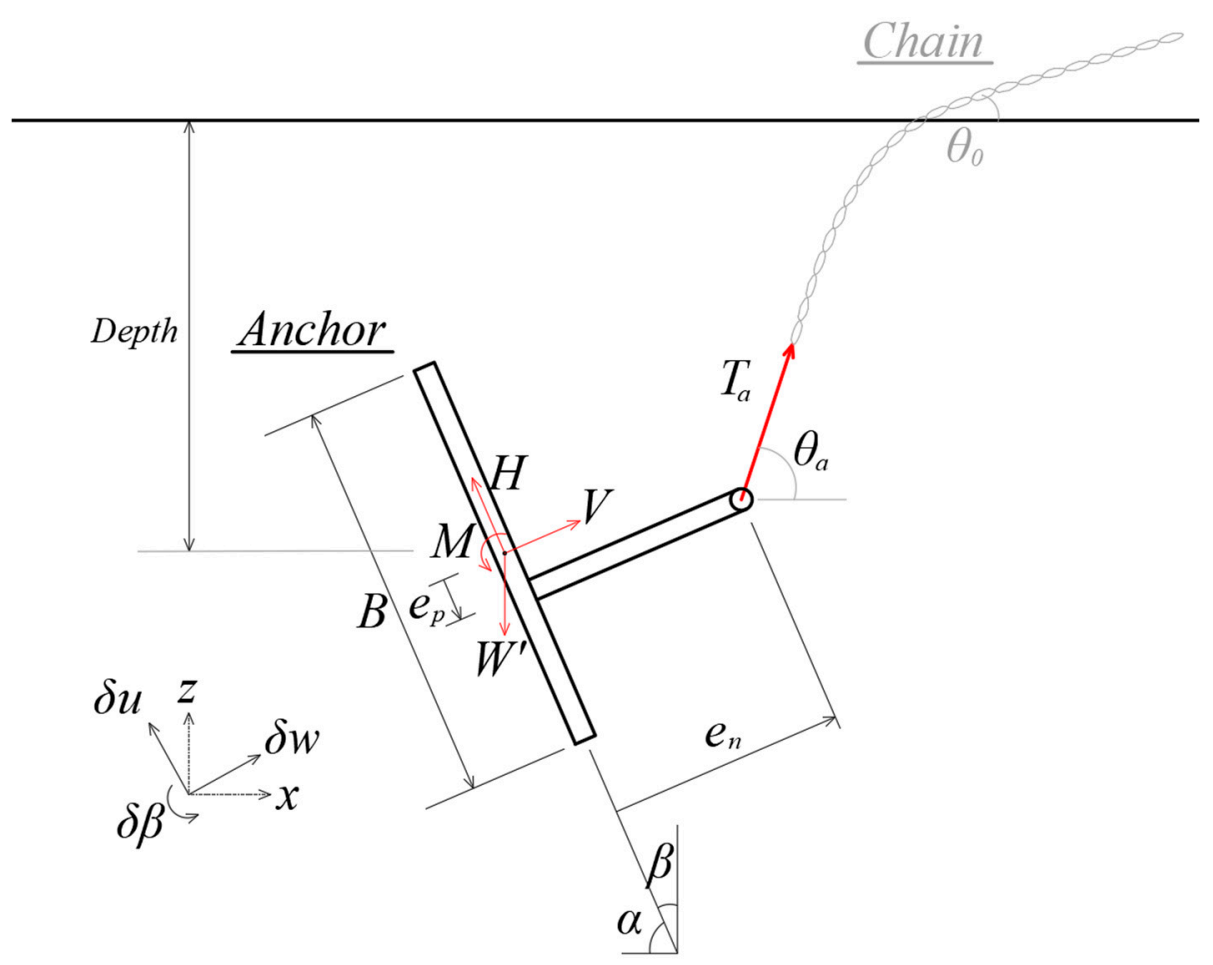

Figure 1. Schematic two-dimensional representation of the anchor and chain geometry and definition of force, displacement and geometrical variables.

The overall in-plane length of the anchor's fluke is denoted by $B$, while the position (eccentricity) of the padeye with respect to the centre of the fluke is defined by $e_{p}$ and $e_{n}$ in the direction parallel and perpendicular to the fluke, respectively. The chain is connected to the padeye of the anchor exerting a force $T_{a}$ with an inclination $\theta_{a}$, while the inclination of the chain at the mudline is denoted by $\theta_{0}$. The pulling force of the chain results in a combination of loads to the anchor: normal $(V)$, sliding $(H)$ and rotational $(M)$ forces which are considered to be applied at the centre of the fluke (i.e., $B / 2$ from each end of the anchor). For circular anchors, $B$ is taken is the diameter of the plate. Imposing force equilibrium conditions to the anchor, the following relationships can be obtained:

$$
\begin{gathered}
V=T_{a} \sin \left(\beta+\pi / 2-\theta_{a}\right)-W / \sin \beta \\
H=T_{a} \cos \left(\beta+\pi / 2-\theta_{a}\right)-W / \cos \beta \\
M=T_{a}\left[e_{n} \cos \left(\beta+\pi / 2-\theta_{a}\right)+e_{p} \sin \left(\beta+\pi / 2-\theta_{a}\right)\right.
\end{gathered}
$$

where $\beta$ is the current inclination of the anchor from the vertical direction and $W^{\prime}$ is the effective anchor weight. The incremental tangential and normal displacement (with respect to the fluke direction) and rotation of the anchor at the centre of the fluke are defined by $\delta u$, 
$\delta w$ and $\delta \beta$. The horizontal and vertical displacement increments with respect to the global coordinate system, defined by $\delta x$ and $\delta z$, are linked to the incremental displacement by

$$
\begin{aligned}
& \delta x=\cos (\beta) \delta w-\sin (\beta) \delta u \\
& \delta z=\sin (\beta) \delta w+\cos (\beta) \delta u
\end{aligned}
$$

The local coordinate system given by $\delta w$ and $\delta u$ moves along with the anchor padeye, while the global coordinate system is fixed and has its origin on the initial position of the anchor padeye. It is assumed that the incremental displacements $\delta u$ and $\delta w$ are defined with respect to the current orientation $\beta$, following the formulation by Cassidy et al. [4].

\subsection{Modelling Strategy}

A visual representation of the adopted modelling strategy is shown in Figure 2. The approach combines a macro-element for the anchors with a simple one-dimensional undrained shearing and consolidation model for a soil element representative of the whole soil mass. The macro-element model governs the force-displacement behaviour and kinematics of the anchor, while the one-dimensional undrained shearing and consolidation model for the soil tracks the evolution of pore water pressure generation, effective stress and soil density which govern the current strength of the soil on which the capacity of the anchor depends. Since the loading and failure of an anchor are closely related to shear stress mobilisation and shear failure in the soil around of the anchor, a simple onedimensional shearing condition was selected as the stress path for the representative soil element. This soil element allows for estimating the amount of shear stress mobilised in the soil, which governs the shear-induced excess pore water pressure generation, as a function of the anchor loading. The two model components will be discussed in the following Sections 2.3 and 2.4, respectively. The two models are linked by some compatibility conditions which, among others, impose that the mobilised capacity in the anchor is equal to the mobilised strength in the soil. This will be further discussed in Section 2.5.

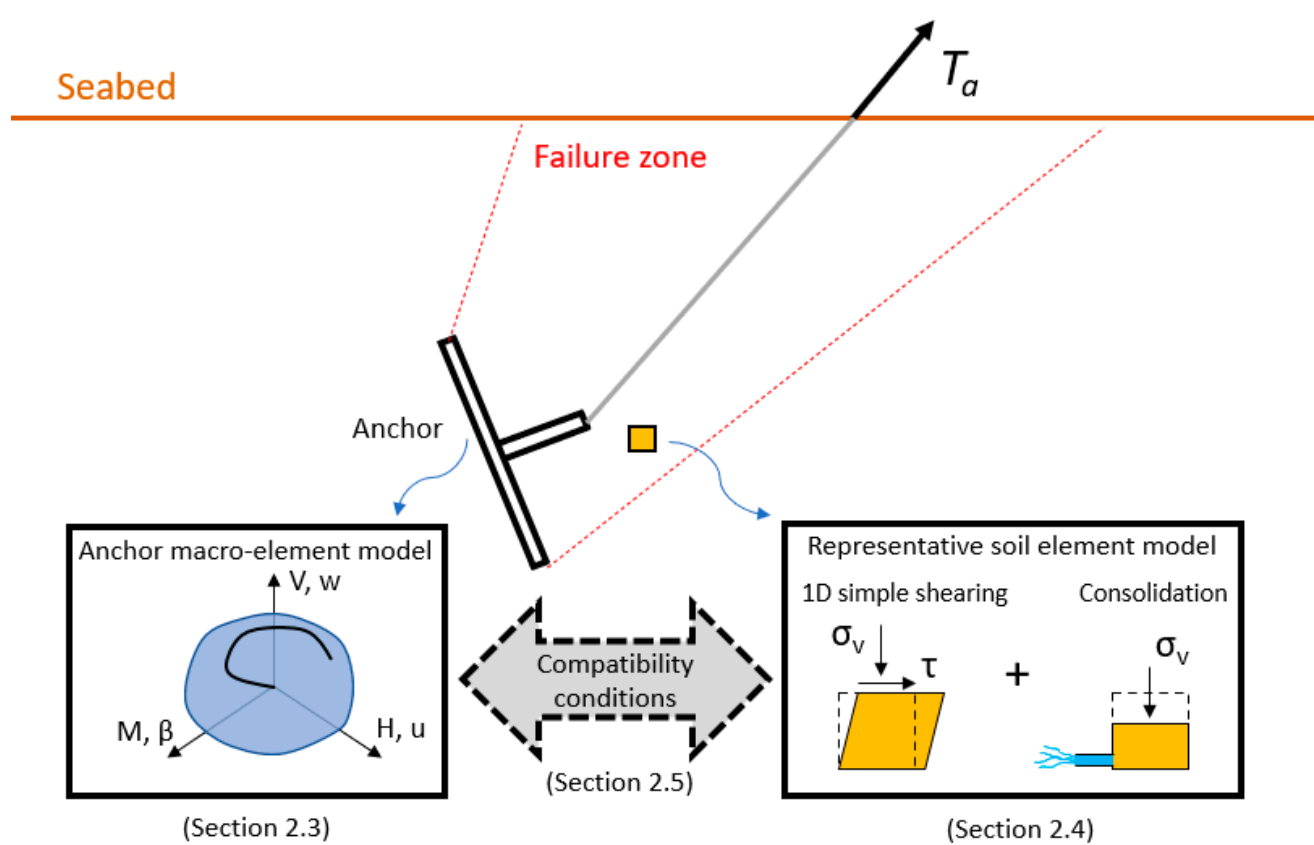

Figure 2. Relationship between the macro-element model for the anchor and the soil constitutive model.

It should be clarified that the representative soil element is an idealised concept and its location with respect to the anchor is unknown. However, since the anchor's capacity is typically related to the undrained shear strength at the depth of the anchor mid-point, it is 
initially assumed that the representative soil element lies at such depth. Nevertheless, this condition can be easily altered if required in future developments of the model.

\subsection{Macro-Element Model for the Anchor}

The kinematics of the anchor are given by a previously published macro-element model [27]. A schematic view of the surfaces adopted in this macro-element model is presented in Figure 3 in a simplified two-dimensional representation, but it should be reminded that the model is formulated in a three-dimensional loading space $(H, V, M)$.

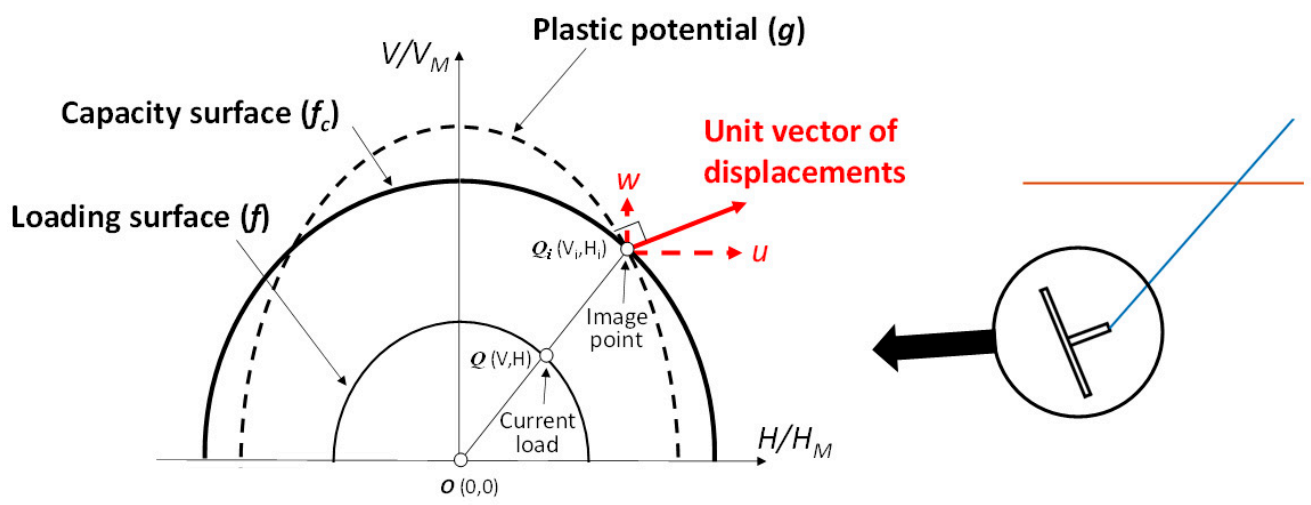

Figure 3. Schematic two-dimensional representation $\left(M / M_{M}=0\right)$ of the model surfaces, force state and plastic potential introduced in the proposed macro-element model for the plate anchor.

The current load (point $Q$ ) lies always on the loading surface which follows the shape proposed by Bransby and O'Neill [28] and employed by several other researchers $[4,5,29-32]$. The size of the loading surface is governed by the hardening parameter $\rho_{c}$ which varies between 0 and 1 :

$$
f=\left(\frac{V}{V_{M}}\right)^{q}+\left(\frac{|M|}{M_{M}}\right)^{m}+\left(\frac{|H|}{H_{M}}\right)^{n}-\rho_{c}=0
$$

where $V_{M}, H_{M}$ and $M_{M}$ are the normal, sliding and rotational capacity when acting independently on the anchor; $m, n$ and $q$ are exponents that define the shape of the loading surface in the $V / V_{M} / H / H_{M} / M / M_{M}$. The capacities $V_{M}, H_{M}$ and $M_{M}$ are commonly defined through the capacity factors $N_{v}=V_{M} /\left(A_{p} \tau_{c}\right), N_{h}=H_{M} /\left(A_{p} \tau_{c}\right)$ and $N_{m}=M_{M} /\left(A_{p} B \tau_{c}\right)$, where $A_{p}$ is the area of the plate and $\tau_{c}$ is the available shear strength of the soil. The capacity surface $\left(f_{c}\right)$ is obtained for a value of $\rho_{c}=1$.

Within the framework of incremental plasticity theory, the size of the loading surface is linked to the plastic displacement $d_{a}$ following a hardening rule analogous to that proposed by Nova and Montrasio [12] but expressed in differential form:

$$
\delta \rho_{c}=R_{0} \delta d_{a}
$$

where $R_{0}$ is the non-dimensional hardening parameter. The total incremental displacement $\delta d_{a}$ is defined as the resultant of the increments of normal $(\delta w)$, tangential $(\delta u)$ and rotational $(\delta \beta)$ displacements:

$$
\delta d_{a}=\sqrt{(\delta w)^{2}+(\delta u)^{2}+(B \delta \beta)^{2}}
$$

The vector of anchor incremental displacements $\delta q(\delta w, \delta u$ and $\delta \beta)$ is given by the flow rule presented in Equation (9):

$$
\delta q=\left(\begin{array}{c}
\delta w \\
\delta u \\
B \delta \beta
\end{array}\right)=\mu\left(\begin{array}{c}
\partial g / \partial V \\
\partial g / \partial H \\
\partial g / \partial(M / B)
\end{array}\right)
$$

where $g$ is the non-associative plastic potential surface and $\mu$ is the plastic multiplier. 
The plastic potential is calculated on the image point on the capacity surface (point $Q_{i}$ ), defined through a radial mapping rule passing through the current load (point $Q$ ) from the origin (point $O$ ), as shown in Figure 3. The expression for the plastic potential surface is similar to that of the loading surface, but its shape is modified by the scaling factors $\xi, \chi$ and $\omega$ which modify the skewing of the surface with the normal, sliding and rotational capacities $\left(V_{M}, H_{M}\right.$ and $M_{M}$, respectively):

$$
g=\left(\frac{V_{i}}{V_{M} / \xi}\right)^{q}+\left(\frac{\left|M_{i}\right|}{M_{M} / \omega}\right)^{m}+\left(\frac{\left|H_{i}\right|}{H_{M} / \chi}\right)^{m}-\rho_{g}=0
$$

where $V_{i}, H_{i}$ and $M_{i}$ define the image point on the bounding surface (point B in Figure 3).

The expression of the plastic multiplier $\mu$ is the same for all displacement components in Equation (9), but their relative values can be determined through the ratios of displacements to one another, defined as follows [5]:

$$
\begin{gathered}
\frac{\delta w}{\delta \beta}=\frac{\partial g / \partial V}{\partial g / \partial(M / B)} \\
\frac{\delta u}{B \delta \beta}=\frac{\partial g / \partial H}{\partial g / \partial(M / B)}
\end{gathered}
$$

The consistency condition (Equation (13)) ensures that the current load state $\left(Q=[V, H, M]^{\mathrm{T}}\right)$ lies always on the loading surface.

$$
d f\left(Q, \rho_{c}\right)=\frac{\partial f}{\partial Q} d Q+\frac{\partial f}{\partial \rho_{c}} d \rho_{c}=0
$$

The macro-element model has been expanded to account for the effects of plate anchor kinematics under cyclic loading [26]. This can be achieved by modifying the hardening rule in order to account for reversal of the loading direction during cyclic loading.

The extension of the monotonic hardening rule in Equation (7) to cyclic conditions is reported in Equation (14) and schematically shown in Figure 4. Equation (14) considers a modified origin which takes into account the load reversal point and a different target value at large displacements, depending on the loading condition: 1 for loading and -1 for unloading. It is worth noting that negative values of $\rho_{c}$ are not physically possible and the curve is drawn in Figure 4 only to show the rationale and methodology for the extension from monotonic to cyclic loading. The expression of the hardening term $\rho_{c, i}$ for the generic loading stage $i$ (where $i$ tracks the cumulative number of applied loading and unloading stages) follows an exponential form analogous to Equation (7) but modified to account for an offset related to the point of load reversal:

$$
\delta \rho_{c, i}=\left( \pm 1-\rho_{c R, i-1}\right) R_{0} \exp \left[-R_{0}\left(d_{a}-d_{a, R, i-1}\right)\right] \delta d_{a}
$$

where the sign (+) holds for loading conditions and the sign (-) for unloading. The quantities $\rho_{c R, i-1}$ and $d_{a, R, i-1}$ are the values of $\rho_{c}$ and $d_{a}$ at the end of the previous loading package (LP), i.e., the point of load reversal. Figure 4 schematically shows the evolution of the term $\rho_{c}$ and the values of the quantities $\rho_{c R, i-1}$ and $d_{a, R, i-1}$ for six loading/unloading cyclic packages followed by monotonic loading to failure.

Extension to cyclic loading of the incremental displacement definition is automatically captured by the flow rule defined in Equation (9), since the radial mapping rule automatically considers the flow direction on the opposite side of the load reversal point. 


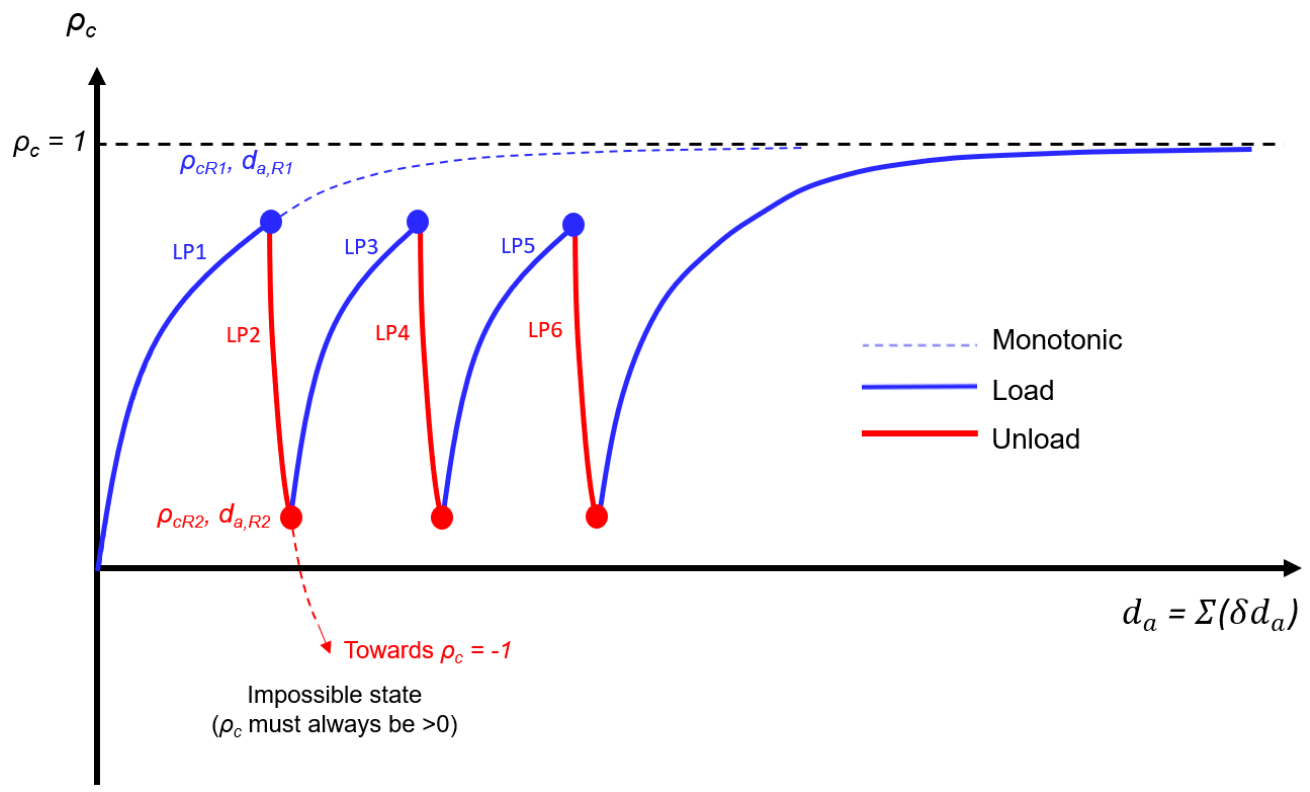

Figure 4. Representation of the hardening term $\rho_{c}$ under unloading-reloading behaviour.

\subsection{One-Dimensional Shear and Consolidation Model for Representative Soil Element}

In order to simplify the modelling of the soil behaviour, the processes of shearing and consolidation are decoupled. It is assumed that undrained shearing of the soil is triggered by the loading of the anchor, while consolidation is triggered by the process of pore water pressure dissipation with time during stages of maintained anchor loading or load application over a period of time. Partial drainage is therefore simulated through the application of fully undrained shear induced by the load followed by pure consolidation during a period of time equal to the duration of the load. This follows a similar approach to that employed in other studies, where partial drainage was simulated through a series of undrained loading phases followed by consolidation phases during which the load was kept constant [19,33-35]. Fundamental model parameters are shared between the consolidation and shearing mechanisms as detailed in the following.

\subsubsection{One-Dimensional Consolidation Model}

The one-dimensional consolidation model for the soil follows the conventional yielding assumed for clays under one-dimensional compression [36]. As shown in Figure 5, the model considers the existence of a normal compression line (NCL) and an unloadingreloading line (URL) which are both linear in the specific volume $(v)$ versus $\ln \sigma^{\prime}$ plane. The equation of the NCL can be expressed as

$$
v_{N C L}=\Gamma_{N C L}-\lambda \ln \sigma \prime
$$

where $\Gamma_{N C L}$ is the intercept on the $v$ axis for $\sigma^{\prime}=1 \mathrm{kPa}$ and $\lambda$ is its slope. The slope of the URL in the $v-\ln \sigma^{\prime}$ plane is defined by $\kappa$.

The process of consolidation is governed by the dissipation of the pore water pressure previously generated by the undrained shearing. Several expressions can be employed to model the pore water pressure dissipation. In this work, the hyperbolic relationship suggested by Singh and Ramaswamy [22] for plate anchors is adopted:

$$
\frac{\Delta u}{\Delta u_{\text {initial }}}=\frac{1}{1+\left(\frac{T}{T_{50}}\right)^{a}}
$$

where $\Delta u / \Delta u_{\text {initial }}$ is the normalised excess pore pressure, $a$ is a curve fitting parameter and $T_{50}$ is the dimensionless time factor $T$ for $50 \%$ dissipation of the initial excess pore pressure. 
The factor $T$ is given by Equation (16), where $c_{v}$ is the coefficient of consolidation and $t_{c}$ is the time of consolidation.

$$
T=c_{v} t_{c} / B^{2}
$$

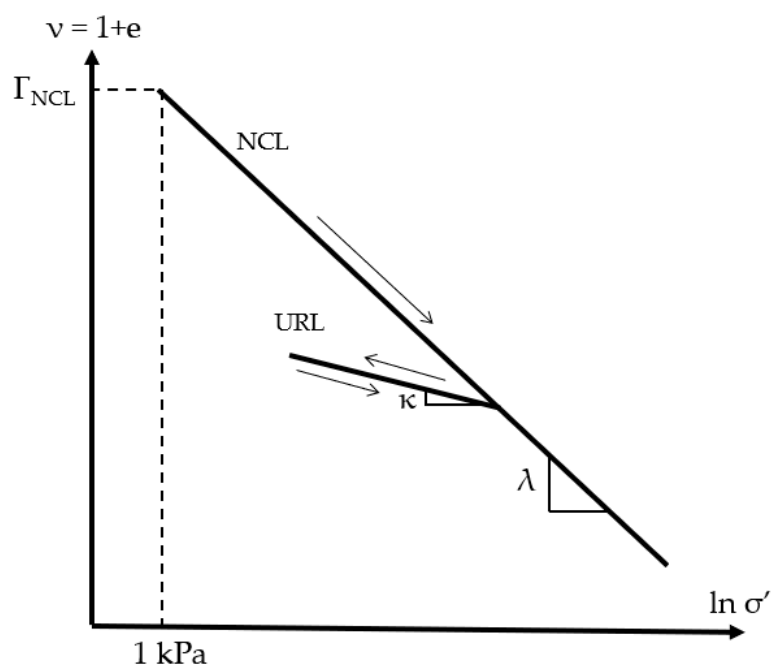

Figure 5. Normal compression line (NCL) and unloading-reloading line (URL) represented in the specific volume versus vertical effective stress plane.

\subsubsection{One-Dimensional Undrained Shearing Model}

The one-dimensional undrained shearing model builds upon a critical state theory and bounding surface plasticity framework to allow flexibility in model capabilities and the application to a large range of soils from clays to sand. However, the model application to undrained shearing conditions only combined with the unnecessity to track the evolution of soil strains considerably simplifies the required modelling equations as shown later in this section.

A schematic view of the model behaviour is shown in the shear stress $(\tau)$ versus effective stress $\left(\sigma^{\prime}\right)$ plane (Figure 6) and in the specific volume $(v)$ versus $\ln \sigma^{\prime}$ plane (Figure 7). As is typical of direct simple shear tests, the total vertical stress $\sigma$ is assumed to be constant during undrained shearing; therefore, any changes in the vertical effective stress $\sigma^{\prime}$ are assumed to be due to the variation in the pore pressure $u_{w}$. The critical state line (CSL) is linear in the $v$ versus $\ln \sigma^{\prime}$ plane and parallel to the NCL defined in the one-dimensional consolidation model. The equation for the CSL is

$$
v_{C S L}=\Gamma_{C S L}-\lambda \ln \sigma \prime
$$

where $\Gamma_{N C L}$ is the intercept on the $v$ axis for $\sigma^{\prime}=1 \mathrm{kPa}$ of the CSL. In the $\tau-\sigma^{\prime}$ plane, the CSL is also linear and defined by the equation

$$
f_{C S L}(\sigma \prime)=\tau_{C S L}-t \sigma \prime \tan \varphi \prime
$$

where $\varphi /$ is the critical state friction angle and $t$ assumes the value of 1 for loading and -1 for unloading conditions.

The bounding surface defines the current strength and its size is governed by the concept of a state parameter $\psi$ as defined by Been and Jefferies [37]. However, a new form of the state parameter is proposed here to account for the volumetric hardening typically governing the behaviour of cohesive soils:

$$
\psi=\sigma I^{\prime} / \sigma^{\prime} C S
$$

where $\sigma /$ is the current effective stress and $\sigma^{\prime} C S$ is the image point on the critical state line in the $v-\ln \sigma^{\prime}$ plane at the same specific volume. 
LOADING

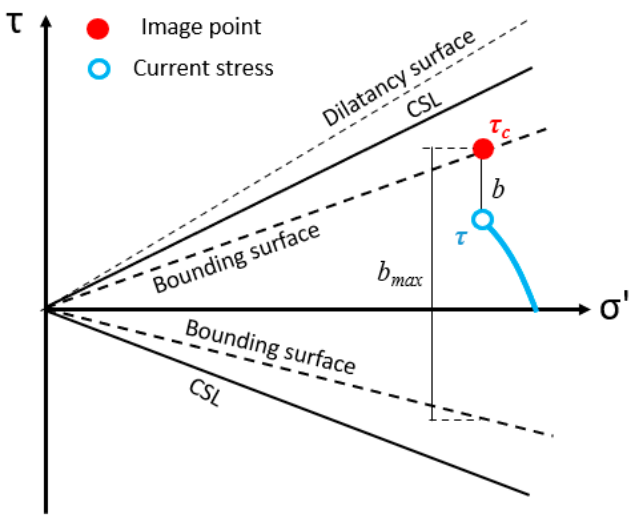

UNLOADING

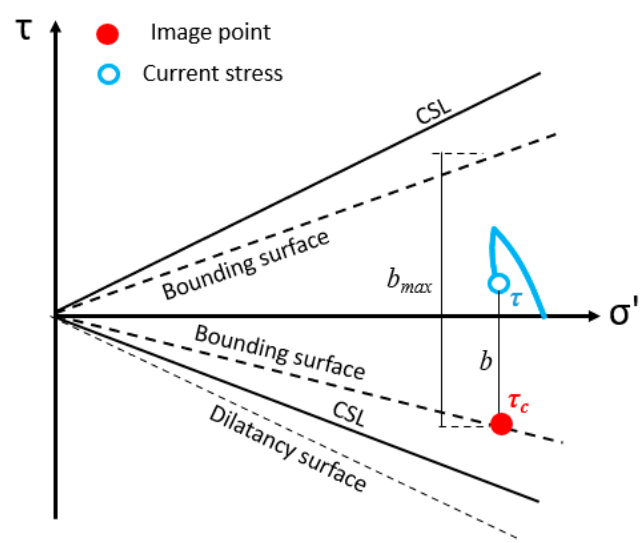

Figure 6. Model for undrained shearing of the representative soil element.

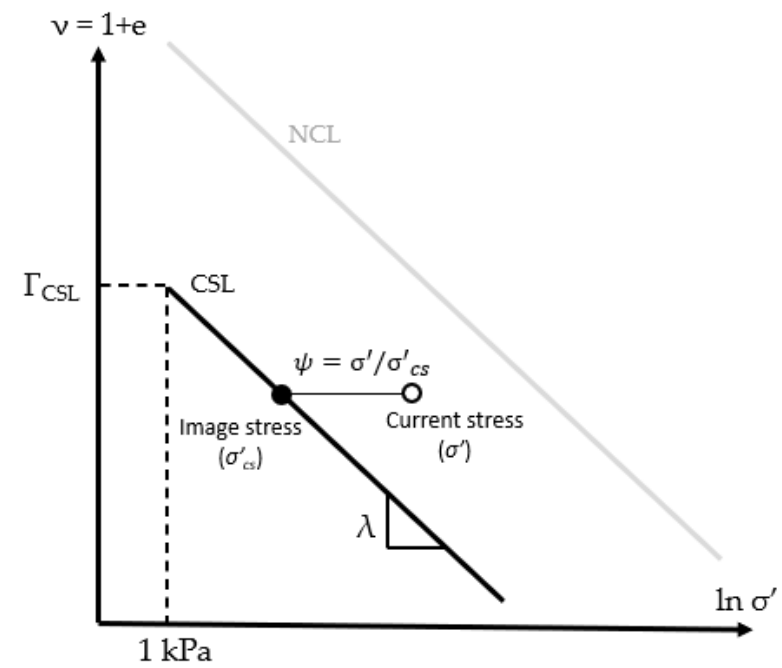

Figure 7. Model for undrained shearing of the representative soil element.

The size of the bounding surface is related to the state parameter as follows:

$$
F(\sigma \prime)=\tau_{c}-t \sigma / \tan \varphi^{\prime \psi^{k_{r}}}
$$

where $k_{r}$ is a model parameter defining the link between the current state parameter and the soil strength.

The stress-dilatancy rule discriminates between soil compression and dilation state and governs the plastic volumetric behaviour during shearing. The form adopted here is similar to that of Cam Clay and to that used by Gajo and Muir Wood [38] which accounts for the effect of the state parameter as proposed by Manzari and Dafalias [39] in order to better capture the effect of picnotropy:

$$
d=A\left[t \tan \varphi / \psi^{k_{d}}-\tau / \sigma \prime\right]
$$

where the parameter $A$ is the flow rule multiplier and the parameter $k_{d}$ governs the dependence of the dilatancy on the state parameter.

The hardening rule is based on deviatoric mapping such as the image stress (or current soil strength, $\tau_{c}$ ) is the vertical projection of the current stress state on the bounding surface such as

$$
\tau_{c}=t \sigma^{\prime} \tan \varphi / \psi^{k_{r}}
$$


The hardening modulus $H_{m}$ is defined in the customary way for bounding surface plasticity models, accounting for a hardening term of the image stress on the bounding surface $\left(H_{b}\right)$ and an arbitrary modulus $\left(H_{f}\right)$ depending on the distance between the current stress state $\tau$ and its image $\tau_{c}$ on the bounding surface:

$$
H_{m}=H_{b}+H_{f}
$$

with

$$
H_{b}=\frac{-t\left(\frac{\tan \left(\varphi^{\prime}\right) \sigma^{\prime} \psi^{k_{r}}}{k_{r}}\right)\left(\frac{\sigma^{\prime} v}{\lambda \exp \left(\frac{\Gamma-v}{\lambda}\right)}\right) m_{\sigma}}{\sqrt{1+\left\{-t \tan \left(\varphi^{\prime}\right) \psi^{k_{r}}\left[1+\frac{\sigma^{\prime} k_{r}}{\psi \exp \left(\frac{\Gamma-v}{\lambda}\right)}\left(1-\frac{\sigma^{\prime} v}{\lambda E}\right)\right]\right\}^{2}}}
$$

where $m_{\sigma}$ is the component parallel to the $\sigma$-axis of the unit vector normal to the plastic potential (defined in Equation (29) below), $E$ is Young's modulus (linked to the bulk modulus $K$ by the assumed Poisson's ratio $v=0.1$ through classic elastic relationships) and

$$
H_{f}=\frac{b^{2}}{C b_{\max }}
$$

where $C$ is a model parameter and $b_{\max }$ is the maximum value that $b$ can assume

$$
b_{\max }=2 \sigma^{\prime} \tan \varphi / \psi^{k_{r}}
$$

The application of the model to undrained shear conditions imposes that $\dot{v}=0$, which implies the following constraint on the volumetric strains-strain relationship:

$$
\dot{\varepsilon}=\frac{m_{\sigma} n_{\tau}}{H_{m}} \dot{\tau}+\left(\frac{1}{K}\right) \dot{\sigma}^{\prime}=0
$$

where $K$ is the elastic bulk modulus of the soil and $n_{\tau}$ is the component parallel to the $\tau$-axis of the unit vector normal to the loading surface:

$$
\begin{gathered}
K=\frac{v \sigma \prime}{\kappa} \\
m_{\sigma}=\frac{t d}{\sqrt{1+d^{2}}} \\
n_{\tau}=\frac{1}{\sqrt{1+\left\{-t \tan \left(\varphi^{\prime}\right) \psi^{k_{r}}\left[1+\frac{\sigma^{\prime} k_{r}}{\psi \exp \left(\frac{\Gamma-v}{\lambda}\right)}\left(1-\frac{\sigma^{\prime} v}{\lambda E}\right)\right]\right\}^{2}}}
\end{gathered}
$$

Rearrangement of Equation (27) provides a direct relationship for the evolution of effective stress during undrained shearing:

$$
\dot{\sigma}^{\prime}=-K \frac{m_{\sigma} n_{\tau}}{H_{m}} \dot{\tau}
$$

The generation of the pore water pressure can be determined using $\dot{\sigma}^{\prime}=\dot{\sigma}-\dot{u}_{w}$, while the currently available soil strength at any moment of loading can be determined using Equation (22).

\subsection{Soil-Anchor Compatibility Conditions}

Careful compatibility conditions must be imposed between the macro-element model for the anchor and soil model to ensure that the two models are closely linked. As discussed above, the current soil strength $\tau_{c}$ governs the capacity surface of the macro-element model through the terms $V_{M}, H_{M}$ and $M_{M}$. The further condition is the assumption that the 
mobilised capacity of the anchor $\rho_{\mathcal{c}}$ corresponds to the mobilised soil strength during undrained shearing:

$$
\rho_{c}=\tau / \tau_{c}
$$

This ensures that both models reach the failure conditions at the same point, such as the anchor capacity is fully governed by the capacity of the soil. Since the function of the representative soil element is to track the changes in excess pore water pressure and the related changes in soil effective stress and available strength, no relationship between the displacements of the anchor and strain in the representative soil element is required in these modelling developments.

\section{Modelling Analysis}

The capabilities and benefits of the newly proposed modelling approach are analysed here for both mono-directional and three-dimensional cyclic loading conditions of the anchor, as schematically shown in Figure 8. The first loading scenario (Figure 8a) builds upon the experimental work by Zhou et al. [25] and the model capabilities in capturing the increase in strength induced by consolidation processes during a cyclic mono-directional is verified. The capabilities of the model are then further explored against a cyclic scenario imposing a three-dimensional loading on the anchor. This is an idealised simulation of a vertically installed anchor subjected to a vertical pull at its padeye similar to a mooring system in offshore applications. The macro-element model is used to obtain insight on the mechanisms affecting the capacity of the anchor.

Seabed level

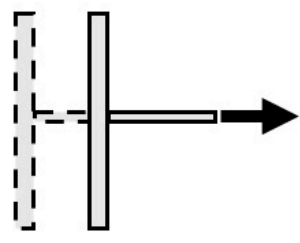

Loading scenario 1

following Zhou et al. [25]

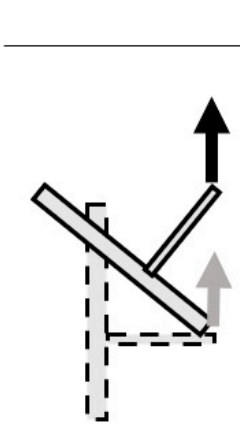

Loading scenario 2 Seabed level

Figure 8. Loading scenarios for the cyclic loading of a plate anchor.

\subsection{Case 1: One-Dimensional Anchor Loading}

\subsubsection{Geometry and Model Parameters}

The first scenario is the benchmarking against the loading conditions shown by Zhou et al. [25]. In this experimental work, a circular plate anchor embedded in a normally consolidated carbonate muddy silt is subjected to long-term cyclic loading. Two tests are considered here: (1) a rapid (undrained) monotonic loading to failure; (2) a cyclic test followed by a rapid monotonic loading to failure.

The cyclic test involved the application of 1080 cycles to the vertical circular plate with a diameter of $B=5.25 \mathrm{~m}$ (prototype scale) installed at an embedment (measured to the centre of the plate) of $4.3 \mathrm{D}$. The frequency of the cycles was $0.4 \mathrm{~Hz}$. The anchor was then horizontally loaded to failure after the cyclic loading. The load on the anchor $T_{a}$ is assumed to be constant during consolidation, similar to a maintained load. Drainage within the cycle is modelled by imposing a consolidation stage after each packet of loading or unloading and for the same duration of the packet. 
Since this experiment only involved forces that are normal to the anchor plane, where no rotation occurs, it follows $V=T_{a}$ and $H=M=0$. This greatly reduces the number of necessary model parameters as indicated in Table 1 . As the only load involved in the test is normal to the anchor plane, it is reasonable to assume that the mobilisation of the anchor capacity $\rho_{\mathrm{c}}$ depends solely on the ratio between the current load $V$ and the normal capacity $V_{M}$; therefore, $q=1$. The normal capacity $N_{v}$ was taken as 9 , based on previous studies for circular plates in clay [40]. Owing to the lack of displacements parallel to the anchor plane or any rotation $(u=0$ and $\beta=0)$, the calibration of the plastic potential parameter is unnecessary in this case. It follows that, for the one-dimensional load case, only the parameter $R_{0}$ needs to be calibrated. A value of $R_{0}=80$ was selected to match the displacement of the anchor during cyclic loading.

Table 1. Geometry and modelling parameters used for Scenario 1.

\begin{tabular}{|c|c|c|c|}
\hline \multicolumn{4}{|c|}{ PARAMETERS FOR ANCHOR } \\
\hline Parameter & Description & Value & Remarks \\
\hline$D$ & Diameter of anchor (m) & 5.25 & Zhou et al. [25] \\
\hline$m$ & Shape of loading surface (moment) & - & \\
\hline$n$ & Shape of loading surface (horizontal) & - & \\
\hline$q$ & Shape of loading surface (vertical) & 1 & \\
\hline$N_{v}$ & Normalised normal capacity factor & 9 & Rowe and Davis [40] \\
\hline$N_{h}$ & Normalised sliding capacity factor & - & \\
\hline$N_{m}$ & Normalised rotational capacity factor & - & \\
\hline$\xi$ & Plastic potential parameter (vertical) & - & \\
\hline$\chi$ & Plastic potential parameter (horizontal) & - & \\
\hline$\omega$ & Plastic potential parameter (moment) & - & \\
\hline$R_{0}$ & Hardening parameter & 80 & Calibrated \\
\hline \multicolumn{4}{|c|}{ PARAMETERS FOR SOIL } \\
\hline$c_{v}$ & Coefficient of consolidation $\left(\mathrm{m}^{2} / \mathrm{h}\right)$ & 11 & \\
\hline$v$ & Poisson's ratio & 0.1 & \\
\hline$\lambda$ & Slope of NCL and CSL & 0.287 & Chow et al. [41] \\
\hline$\kappa$ & Slope of the swelling line & 0.036 & Chow et al. [41] \\
\hline$\Gamma_{N C L}$ & Specific volume at $\sigma_{v}{ }^{\prime}=1 \mathrm{kPa}$ on the NCL & 4.0 & Chow et al. [41] \\
\hline$\varphi_{c S}$ & Critical state friction angle $\left(^{\circ}\right)$ & 40 & Chow et al. [41] \\
\hline$\Gamma_{C S L}$ & Specific volume at $\sigma_{v}^{\prime}=1 \mathrm{kPa}$ on the CSL & 3.8 & \\
\hline$T_{50}$ & $\begin{array}{l}\text { Dimensionless time factor for } 50 \% \\
\text { consolidation }\end{array}$ & 0.02 & \\
\hline$a$ & Consolidation curve fitting & 1.15 & \\
\hline$A$ & Flow rule multiplier & 0.2 & \\
\hline$k_{d}$ & State parameter multiplier in flow rule & 5.0 & \\
\hline$k_{r}$ & Link between state parameter and strength & 2.0 & \\
\hline C & Hyperbolic relationship parameter & 0.0002 & \\
\hline
\end{tabular}

The soil conditions featured a normally consolidated carbonate muddy silt reconstituted from an offshore sediment with an effective unit weight of $\gamma^{\prime}=5.2 \mathrm{kN} / \mathrm{m}^{3}$ and initial moisture content in the range of $65-88 \%$. Based on other centrifuge samples [41] of the same soil used in the anchor tests, the properties of the normal consolidation line (NCL) are summarised in Table 1. The other model parameters for the one-dimensional undrained shearing and consolidation model for the soil element were selected to fit the experimental data.

\subsubsection{Model Predictions}

Figure 9 reports a comparison between the model simulations and the centrifuge data of the monotonic cyclic capacity and the cyclic tests' response. It can be seen that the pre-cyclic monotonic capacity (test 1 ) as well as the post-cyclic capacity (test 2) of the anchor is well captured by the model. For the initial proof loading, $q_{u}=489 \mathrm{kPa}$ is observed in the centrifuge test, whereas $q_{u}=514 \mathrm{kPa}$ is obtained from the model simulation. For the post-cyclic capacity, a value of $q_{u}=737 \mathrm{kPa}$ is observed for the centrifuge tests, whereas $q_{u}=743 \mathrm{kPa}$ is obtained for the macro-element model simulation. Furthermore, good 
agreement can be seen between the displacements at which the peak load is reached (x/D) - 1.4 for the centrifuge test and 1.15 for the model prediction. The less satisfactory performance in predicting the anchor's stiffness can be achieved through modification of the adopted hardening relationship in Equation (12), but this is outside the scope of the current paper and will be addressed in future work.

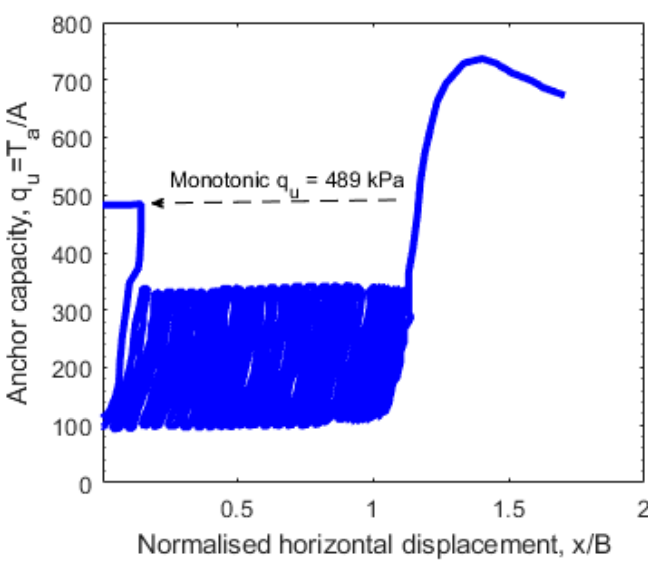

(a)

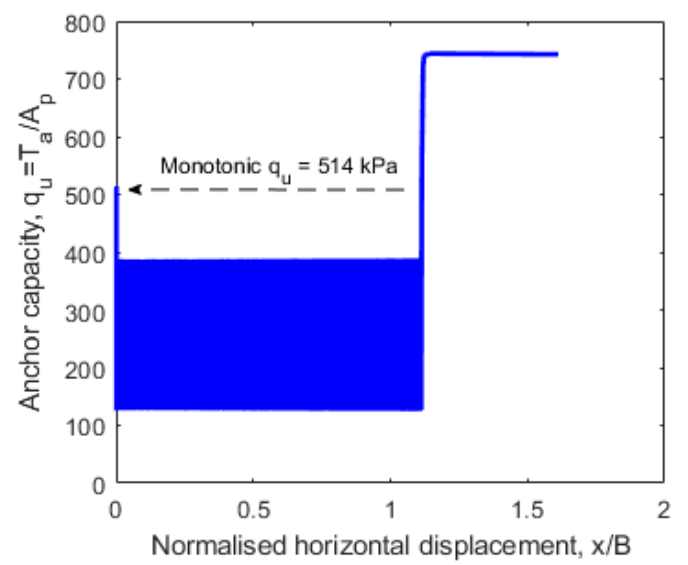

(b)

Figure 9. Comparison between experimental and simulated anchor capacities: (a) centrifuge test [25]; (b) macro-element model result.

The analysis of the soil element response can provide an insight on the observed behaviour. Figure 10 displays the evolution of excess pore water pressure during the simulation of the cyclic tests. An excess pore pressure of $23 \mathrm{kPa}$ is generated during the initial monotonic loading. As cyclic loading is applied, the excess pore pressure increases up to $44 \mathrm{kPa}$, after which the pore pressure dissipation due to soil consolidation outweighs the pore pressure generation. The pore pressure decreases to $12 \mathrm{kPa}$ after 1080 cycles, followed by an extra pore pressure increase up to $18 \mathrm{kPa}$ during the post-cyclic pull-out. Therefrom, the vertical effective stress $\sigma_{v}{ }^{\prime}$ after cycles is higher than after monotonic pullout, and consequently, the soil's shear stress-and hence the anchor capacity-is higher. This can be visualised in Figure 11, which shows the effective stress path in terms of specific volume (Figure 11a) and shear stress (Figure 11b) versus vertical effective stress. As discussed in Zhou et al. [25], the account for changes in soil is of major importance in the cyclic assessment of the anchor behaviour.

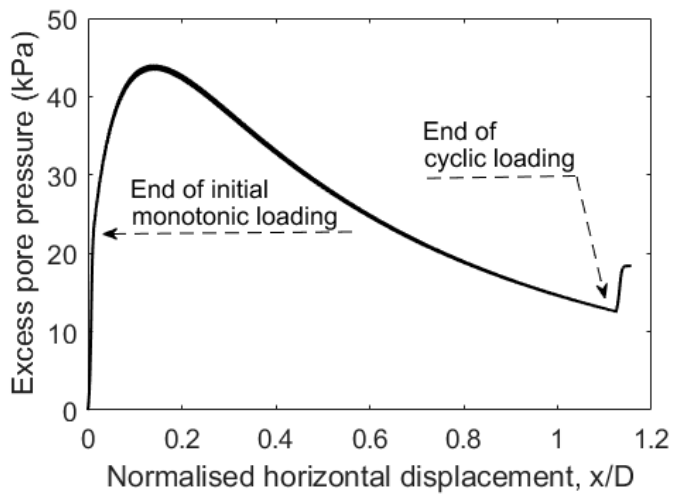

Figure 10. Excess pore water pressure generation during cyclic loading of the circular plate anchor. 


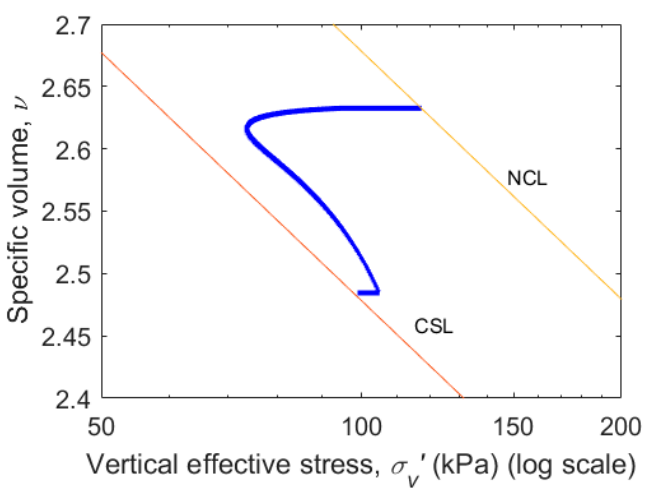

(a)

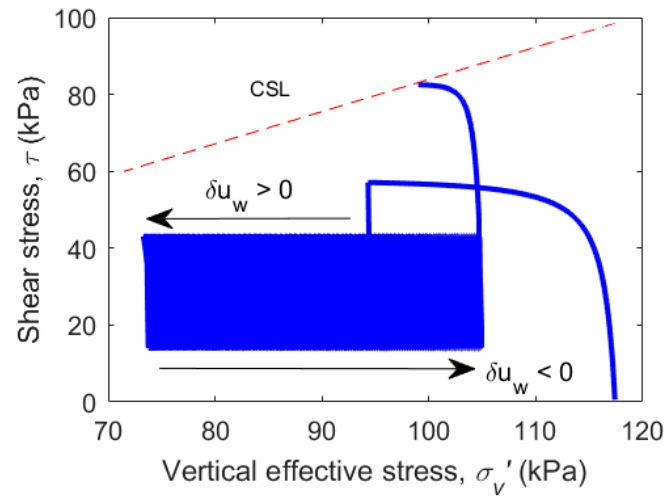

(b)

Figure 11. Evolution of soil strength: (a) $v-\sigma_{v}{ }^{\prime}$ plane; (b) $\tau-\sigma_{v}{ }^{\prime}$ plane.

\subsection{Case 2: Three-Dimensional Anchor Loading}

\subsubsection{Geometry and Model Parameters}

The second loading scenario aims to evaluate the model capabilities for a configuration in which the three-dimensional capacity of the plate anchor is mobilised. The configuration follows the study performed by Cassidy et al. [4], but the assessment is extended here to the cyclic capacity of the anchor. The geometry considers a vertical plate anchor pulled vertically at its padeye, featuring both a horizontal and a vertical eccentricity with respect to the plate anchor mid-point, as shown in Figure 8b.

The dimensions of the rectangular anchor are $B=4.64 \mathrm{~m}$ (breadth) and $L=7.92 \mathrm{~m}$ (length), and the anchor padeye is located at an eccentricity of $e_{n}=2.59 \mathrm{~m}$ and an offset of $e_{p}=0.492 \mathrm{~m}$. The initial embedment of the anchor is $20.25 \mathrm{~m}$ which corresponds to an embedment ratio of approximately 4.4. The monotonic uplift of such anchor has been already considered by Peccin da Silva et al. [26], who used the macro-element model for the plate anchor but neglected the evolution of pore pressure generation and soil strength during loading. Peccin da Silva et al. [27] also provided a detailed account for the determination and/or calibration procedure of the anchor macro-element model parameters and the same values are used here, as summarised in Table 2. It should be emphasised that while 10 parameters are employed, the values of the bearing capacity factors $\left(N_{v}, N_{h}\right.$ and $\left.N_{m}\right)$ and shape parameters of the loading surface $f(m, n$ and $q)$ can be assumed based on the previous literature (e.g., [4,28,30,42]). Peccin da Silva et al. [27] demonstrated that two of the plastic potential parameters $(\xi$ and $\chi$ ) typically assume the same value, while the parameters $\omega$ and $R_{0}$ can be calibrated from a monotonic load displacement curve of an anchor. In absence of specific soil data for this idealised loading geometry and scenario, the same soil parameters identified in scenario 1 are employed.

The assessment of the cyclic capacity of the anchor explored both the effect of the duration of cyclic loading (number of cycles) and the loading frequency. The applied amplitude of the cyclic loading was kept fixed in this assessment and forces $T_{a}$ between $10 \%$ and $60 \%$ of the monotonic undrained anchor capacity were applied at the anchor's padeye. 
Table 2. Geometry and modelling parameters for the anchor

\begin{tabular}{|c|c|c|c|}
\hline Parameter & Description & Value & Remarks \\
\hline$B$ & Anchor breadth (m) & 4.64 & Cassidy et al. [4] \\
\hline$L$ & Anchor width (m) & 7.92 & Cassidy et al. [4] \\
\hline$e_{n}$ & Padeye normal eccentricity (m) & 2.59 & Cassidy et al. [4] \\
\hline$e_{p}$ & Padeye offset $(\mathrm{m})$ & 0.492 & Cassidy et al. [4] \\
\hline$m$ & Shape of loading surface (moment) & 2 & Peccin da Silva et al. [27] \\
\hline$n$ & Shape of loading surface (horizontal) & 4 & Peccin da Silva et al. [27] \\
\hline$q$ & Shape of loading surface (vertical) & 4 & Peccin da Silva et al. [27] \\
\hline$N_{v}$ & Normalised normal capacity factor & 14 & Peccin da Silva et al. [27] \\
\hline$N_{h}$ & Normalised sliding capacity factor & 3 & Peccin da Silva et al. [27] \\
\hline$N_{m}$ & Normalised rotational capacity factor & 2 & Peccin da Silva et al. [27] \\
\hline$\xi$ & Plastic potential parameter (vertical) & 1.6 & Peccin da Silva et al. [27] \\
\hline$\chi$ & Plastic potential parameter (horizontal) & 1.1 & Peccin da Silva et al. [27] \\
\hline$\omega$ & Plastic potential parameter (moment) & 1.5 & Peccin da Silva et al. [27] \\
\hline$R_{0}$ & Hardening parameter & 1.0 & Peccin da Silva et al. [27] \\
\hline \multicolumn{4}{|c|}{ PARAMETERS FOR SOIL } \\
\hline$c_{v}$ & Coefficient of consolidation $\left(\mathrm{m}^{2} / \mathrm{h}\right)$ & 11 & From Case 1 \\
\hline$\lambda$ & Slope of NCL and CSL & 0.287 & From Case 1 \\
\hline$\kappa$ & Slope of the swelling line & 0.036 & From Case 1 \\
\hline$\Gamma_{N C L}$ & Specific volume at $\sigma_{v}{ }^{\prime}=1 \mathrm{kPa}$ on the NCL & 4.0 & From Case 1 \\
\hline$\Phi_{C S}$ & Critical state friction angle $\left({ }^{\circ}\right)$ & 40 & From Case 1 \\
\hline$\Gamma_{C S L}$ & Specific volume at $\sigma_{v}{ }^{\prime}=1 \mathrm{kPa}$ on the CSL & 3.8 & From Case 1 \\
\hline$T_{50}$ & $\begin{array}{l}\text { Dimensionless time factor for } 50 \% \\
\text { consolidation }\end{array}$ & 0.02 & From Case 1 \\
\hline$a$ & Consolidation curve fitting & 1.15 & From Case 1 \\
\hline$A$ & Flow rule multiplier & 0.2 & From Case 1 \\
\hline$k_{d}$ & State parameter multiplier in flow rule & 5.0 & From Case 1 \\
\hline$k_{r}$ & Link between state parameter and strength & 2.0 & From Case 1 \\
\hline C & Hyperbolic relationship parameter & 0.0002 & From Case 1 \\
\hline
\end{tabular}

\subsubsection{Model Predictions}

Figure 12 compares the simulations for monotonic undrained loading with those of 10 cyclic tests performed at a frequency of $0.4 \mathrm{~Hz}$. In order to assess the importance of the developments proposed here but also to gain an insight on the cyclic behaviour, the cyclic simulations were performed with and without the account for the behaviour of the representative soil element. When only the anchor kinematics are considered (i.e., neglecting the evolution of soil strength), the post-cyclic capacity of the anchor decreases by approximately $7 \%$ after 10 cycles in comparison with an undrained monotonic pull-out. On the other hand, when the changes in soil strength due to pore water pressure generation and dissipation are considered, the post-cyclic capacity is less than $2 \%$ smaller than the monotonic analysis, but the peak load is also reached for a higher loss of embedment. This increase in anchor capacity when changes in soil strength are considered is due to the pore pressure dissipation exceeding pore pressure generation after a certain number of cycles, making the soil regain part of the vertical effective stress that was lost during the first cycles.

It has been shown [26] that anchor reorientation decreases the anchor post-cyclic capacity, and that the higher the number of cycles, the higher the loss in capacity. On the other hand, it has also been shown [25] that higher numbers of cycles cause a more significant gain in soil strength. Model simulations for a range of number of cycles are provided in Figure 13. Figure 13a,c display the force-displacement and rotational behaviour for the anchor when the changes in soil strength are considered, whereas Figure 13b,d present results for when the soil strength is assumed as constant. The chain load in Figure 13c,d was normalised by the current strength for each step during the model simulations. 


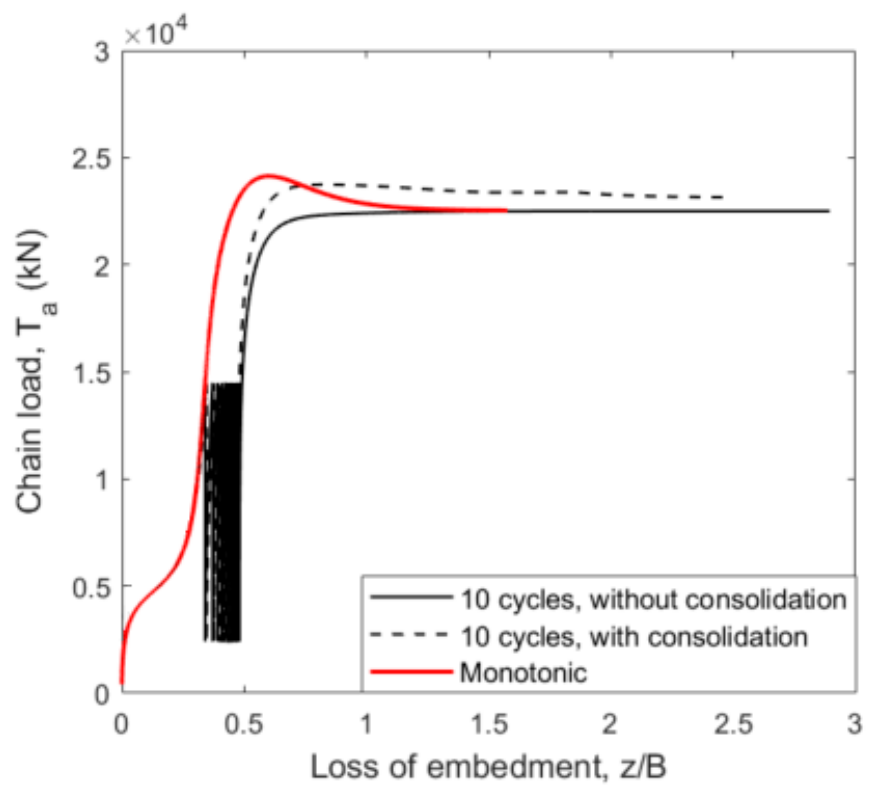

Figure 12. Effect of the evolution of soil strength during 10 cycles of cyclic loading with a frequency of $0.4 \mathrm{~Hz}$.
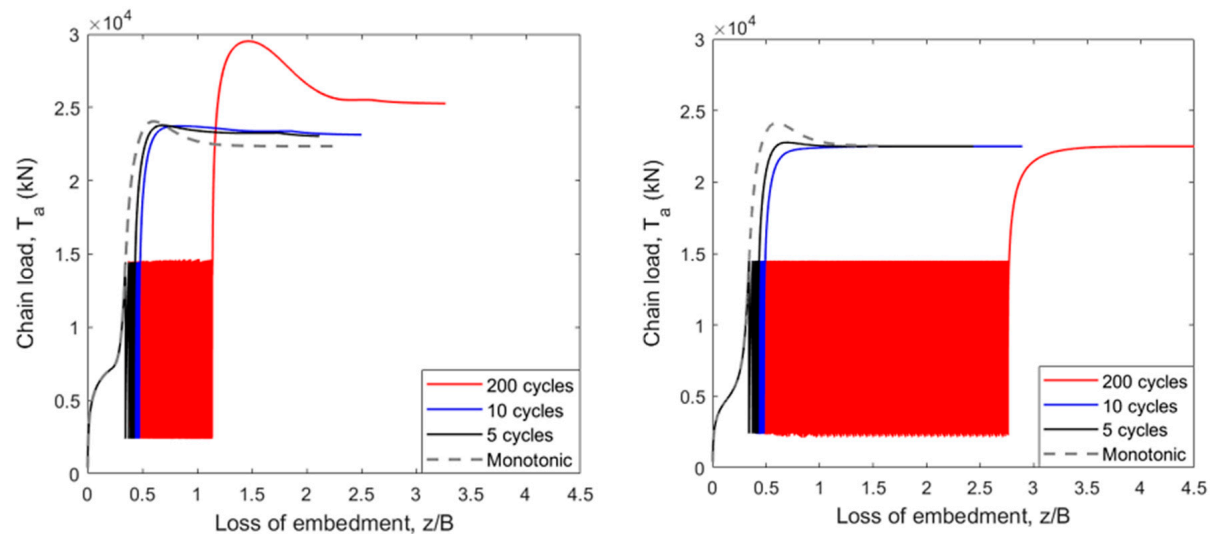

(a)

(b)

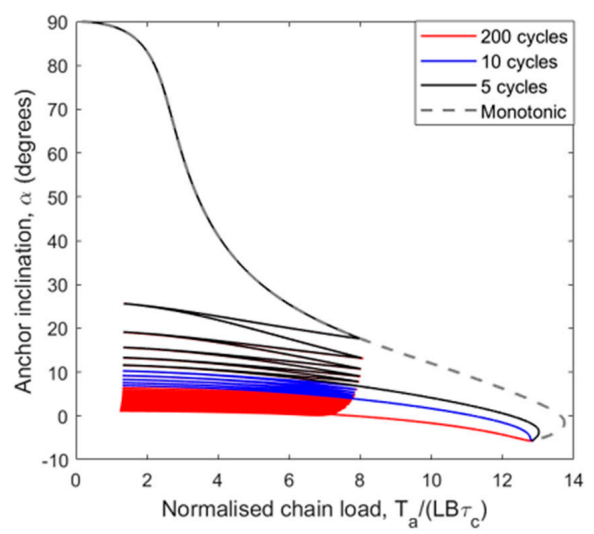

(c)

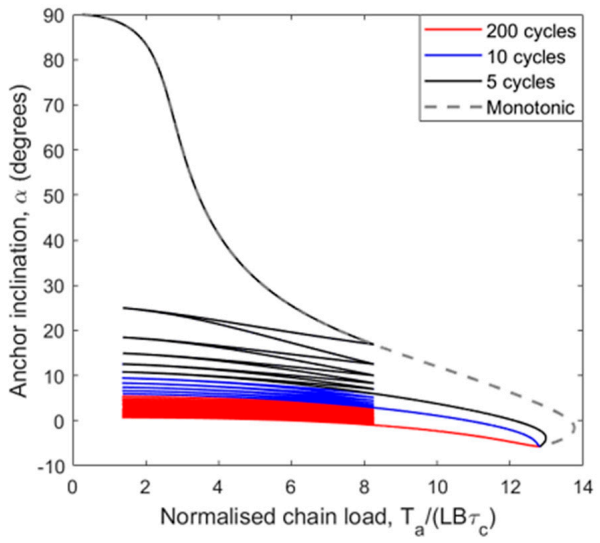

(d)

Figure 13. Effect of the number of cycles on anchor capacity (a) with and (b) without consolidation and on anchor rotation $(\mathbf{c})$ with and $(\mathbf{d})$ without consolidation.

The results in Figure 13b show that, if the soil strength is assumed constant and unaffected by the applied cyclic and consolidation stages, a decrease in post-cyclic peak capacity if compared to the monotonic capacity can be observed. The decrease is about 
$6 \%$ after 5 cycles and $7 \%$ after 10 cycles, after which the capacity loss seems to stabilise, as the same decrease (7\%) is observed after 200 cycles. Conversely, Figure 13a shows that the slight decrease in the peak post-cyclic capacity observed after 5 and 10 cycles (both equal to approximately $2 \%$ ) is followed by a considerable increase in the post-cyclic capacity equal to $23 \%$ after 200 cycles. This suggests that, while the anchor loses some capacity because of its reorientation during cyclic loading (see evolution of rotation in Figure 13b,d), the gain in soil strength due to the consolidation process can initially counteract and then even largely outweigh the loss of capacity caused by the anchor kinematic in the cyclic loading process.

Finally, the effect of the cyclic frequency on the post-anchor capacity is shown in Figure 14, which reports the anchor capacity normalised by the capacity for the highest frequency $\left(T_{a, \text { ref }}=23150 \mathrm{kN}\right.$ for a frequency of $\left.30 \mathrm{~Hz}\right)$ versus the frequency for 30 applied cycles. As expected, higher capacities are achieved for low frequencies due to the occurring of consolidation processes, while lower capacities are achieved for the high cyclic frequency. This trend is compatible with a published framework for interpreting consolidation effects on the plate anchor capacity [7].

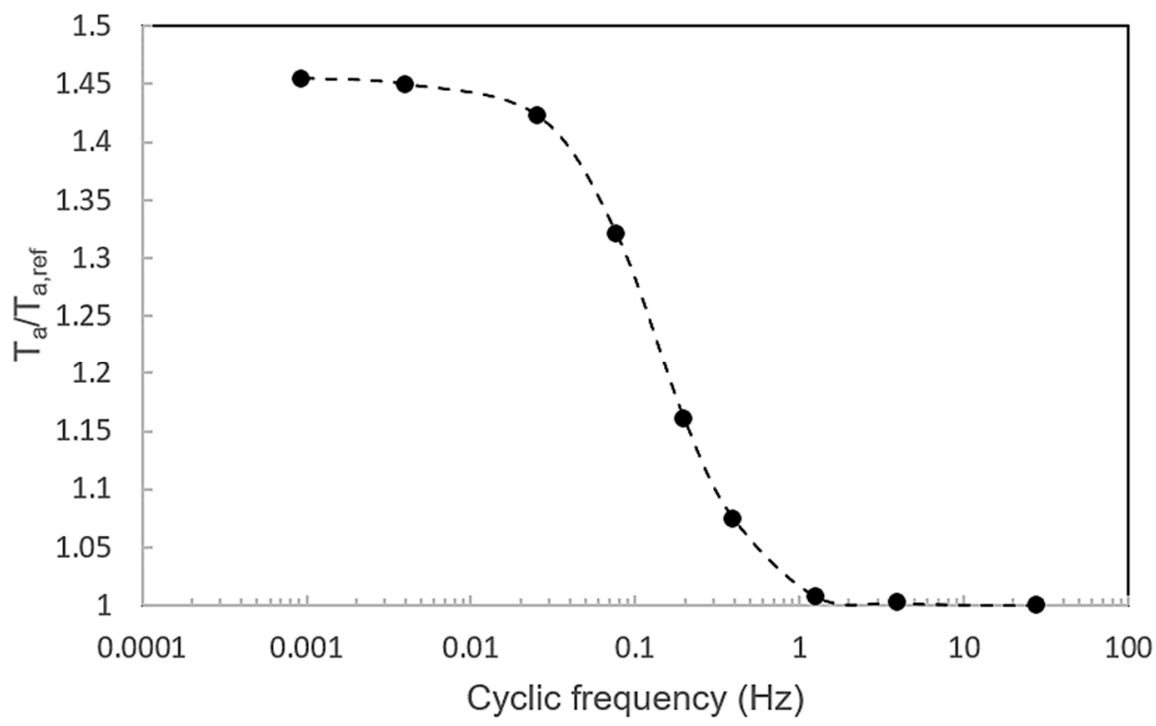

Figure 14. Effect of cyclic frequency on anchor capacity for 30 cycles applied.

\section{Conclusions}

This paper proposed a new macro-element modelling framework aiming to capture the cyclic behaviour of plate anchors and the evolution of the cyclic capacity related to changes in soil strength, as triggered by processes of pore water pressure generation and/or consolidation during loading. The modelling framework combined a macro-element model for the anchor [27] with a one-dimensional model of undrained shearing and consolidation for a soil element, representative of the whole soil mass around the anchor. The onedimensional model of undrained shearing and consolidation for the soil element is based on the critical state and bounding surface concepts in order to accurately capture pore water pressure generation and dissipation during both consolidation and shearing mechanisms.

Capabilities of the model were first demonstrated against one-dimensional loading conditions, featuring a vertical anchor subjected to horizontal loads, reported by Zhou et al. [25]. It was shown that the model can capture the effect of pore water pressure generation and consolidation (simultaneously occurring during cyclic loading of anchors) on the anchor's capacity. Further application of the modelling framework to full threedimensional loading conditions, involving both translation and rotation of the anchor, showed that, during cyclic loading, the anchor capacity may decrease due to the kinematic effect of the anchor reorientation and pore water pressure build-up. However, the gain in soil strength related to the dissipation of pore water pressure generation may counteract 
both detrimental effects and increase the overall cyclic capacity after a certain number of cycles. It is also shown that the higher the number of cycles, the higher the gain in anchor capacity.

Despite being intuitively reasonable, the predictions of the model under threedimensional cyclic anchor loading should be verified through appropriate experimental testing, which is not available to date. Therefore, it is hoped that the results of this paper may provide inspiration for future testing but also provide some indication of the governing mechanism to be considered when assessing the cyclic three-dimensional behaviour of plate anchors. It should be further specified that a detailed calibration procedure for all the parameters of the one-dimensional soil element model could not be developed at present due to the lack of experimental data and it will be the focus of future studies.

Author Contributions: Conceptualization, A.P.d.S., A.D. and D.K.; methodology, A.P.d.S., and A.D.; software, A.P.d.S. and A.D.; validation, A.P.d.S. and A.D.; formal analysis, A.P.d.S., A.D. and S.H.C.; investigation, A.P.d.S., A.D. and S.H.C.; resources, A.P.d.S. and A.D.; data curation, A.P.d.S.; writingoriginal draft preparation, A.P.d.S. and A.D.; writing-review and editing, A.P.d.S., A.D. and S.H.C.; visualization, A.P.d.S. and A.D.; supervision, A.D. and D.K.; project administration, A.P.d.S. and A.D.; funding acquisition, A.P.d.S. and A.D. All authors have read and agreed to the published version of the manuscript.

Funding: This research was funded by University of Bristol, School of Engineering PhD Scholarship. The APC was funded by Partnership for Research in Marine Renewable Energy (PRIMaRE).

Institutional Review Board Statement: Not applicable.

Informed Consent Statement: Not applicable.

Acknowledgments: Not applicable.

Conflicts of Interest: The funders had no role in the design of the study; in the collection, analyses, or interpretation of data; in the writing of the manuscript, or in the decision to publish the results.

\section{References}

1. Europe, W. Floating Offshore Wind Vision Statement; Wind Europe: Brussels, Belgium, 2017.

2. Gaudin, C.; O'Loughlin, C.D.; Bienen, B. Geotechnical modelling for offshore renewables. In Proceedings of the 9th International Conference on Physical Modelling in Geotechnics (ICPMG 2018), London, UK, 17-20 July 2018; CRC Press: Boca Raton, FL, USA, 2018; Volume 1, p. 33.

3. Tian, Y.; Gaudin, C.; Randolph, M.F.; Cassidy, M.J. Influence of padeye offset on bearing capacity of three-dimensional plate anchors. Can. Geotech. J. 2015, 52, 682-693. [CrossRef]

4. Cassidy, M.J.; Gaudin, C.; Randolph, M.F.; Wong, P.C.; Wang, D.; Tian, Y. A plasticity model to assess the keying of plate anchors. Géotechnique 2012, 62, 825-836. [CrossRef]

5. Yang, M.; Aubeny, C.P.; Murff, J.D. Behavior of suction embedded plate anchors during keying process. J. Geotech. Geoenviron. Eng. 2012, 138, 174-183. [CrossRef]

6. Chow, S.H.; O'loughlin, C.D.; Corti, R.; Gaudin, C.; Diambra, A. Drained cyclic capacity of plate anchors in dense sand: Experimental and theoretical observations. Géotechnique Lett. 2015, 5, 80-85. [CrossRef]

7. Chow, S.H.; Diambra, A.; O'Loughlin, C.D.; Gaudin, C.; Randolph, M.F. Consolidation effects on monotonic and cyclic capacity of plate anchors in sand. Géotechnique 2020, 70, 720-731. [CrossRef]

8. Feng, T.; Xu, H.; Song, J.; Zhang, J.; Zhou, M.; Zhang, F. Finite-Element Analysis of Keying Process of Plate Anchors in Three-Layer Soft-Stiff-Soft Clay Deposits. Adv. Civ. Eng. 2019, 1-11. [CrossRef]

9. Muir Wood, D.M. Geotechnical Modelling; CRC Press: Boca Raton, FL, USA, 2003.

10. Skau, K.S.; Grimstad, G.; Page, A.M.; Eiksund, G.R.; Jostad, H.P. A macro-element for integrated time domain analyses representing bucket foundations for offshore wind turbines. Mar. Struct. 2018, 59, 158-178. [CrossRef]

11. Page, A.M.; Grimstad, G.; Eiksund, G.R.; Jostad, H.P. A macro-element pile foundation model for integrated analyses of monopile-based offshore wind turbines. Ocean Eng. 2018, 167, 23-35. [CrossRef]

12. Nova, R.; Montrasio, L. Settlements of shallow foundations on sand. Géotechnique 1991, 41, 243-256. [CrossRef]

13. Gottardi, G.; Houlsby, G.T.; Butterfield, R. Plastic response of circular footings on sand under general planar loading. Géotechnique 1999, 49, 453-469. [CrossRef]

14. Cremer, C.; Pecker, A.; Davenne, L. Cyclic macro-element for soil-structure interaction: Material and geometrical non-linearities. Int. J. Numer. Anal. Methods Geomech. 2001, 25, 1257-1284. [CrossRef] 
15. Gourvenec, S.; Stanier, S.A.; White, D.J.; Morgan, N.; Banimahd, M.; Chen, J. Whole-life assessment of subsea shallow foundation capacity. In Offshore Site Investigation Geotechnics 8th International Conference Proceeding; Society for Underwater Technology: London, UK, 2017; Volume 787, pp. 787-795.

16. Laham, N.I.; Kwa, K.A.; White, D.J.; Gourvenec, S.M. Episodic direct simple shear tests to measure changing strength for whole-life geotechnical design. Géotechnique Lett. 2020, 1-24. [CrossRef]

17. Corti, R.; Gourvenec, S.M.; Randolph, M.F.; Diambra, A. Application of a memory surface model to predict whole-life settlements of a sliding foundation. Comput. Geotech. 2017, 88, 152-163. [CrossRef]

18. Houlsby, G.T.; Cassidy, M.J. A simplified mechanically based model for predicting partially drained behaviour of penetrometers and shallow foundations. Géotechnique Lett. 2011, 1, 65-69. [CrossRef]

19. Flessati, L.; di Prisco, C.; Callea, F. Numerical and theoretical analyses of settlements of strip shallow foundations on normally consolidated clays under partially drained conditions. Géotechnique 2020, 1-21. [CrossRef]

20. Andersen, K.H. Cyclic soil parameters for offshore foundation design. Front. Offshore Geotech. III 2015, 3-82.

21. Yu, H.; Zeng, X.; Li, B.; Lian, J. Centrifuge modeling of offshore wind foundations under earthquake loading. Soil Dyn. Earthq. Eng. 2015, 77, 402-415. [CrossRef]

22. Singh, S.P.; Ramaswamy, S.V. Effect of shape on holding capacity of plate anchors buried in soft soil. J. Geomech. Geoengng 2008, 3, 157-166. [CrossRef]

23. Ponniah, D.A.; Finlay, T.W. Cyclic behaviour of plate anchors. Can. Geotech. J. 1988, 25, 374-381. [CrossRef]

24. Zhou, Z.; White, D.J.; O'Loughlin, C.D. An effective stress framework for estimating penetration resistance accounting for changes in soil strength from maintained load, remoulding and consolidation. Géotechnique 2019, 69, 57-71. [CrossRef]

25. Zhou, Z.; O'Loughlin, C.D.; White, D.J.; Stanier, S.A. Improvements in plate anchor capacity due to cyclic and maintained loads combined with consolidation. Géotechnique 2020, 70, 732-749. [CrossRef]

26. Peccin da Silva, A.; Diambra, A.; Karamitros, D.; Chow, S.H. Macro-element modelling of plate anchor kinematics under cyclic loading in clay. In Proceedings of the International Symposium on Frontiers in Offshore Geotechnics (ISFOG), Austin, TX, USA, 16-19 August 2020.

27. Peccin da Silva, A.; Diambra, A.; Karamitros, D.; Chow, S.H. A non-associative macro-element model for vertical plate anchors in clay. Can. Geotech. J. 2021. [CrossRef]

28. Bransby, M.F.; O'Neill, M. Drag anchor fluke soil interaction in clays. In Proceedings of the 7th International Symposium on Numerical Models in Geomechanics, Graz, Vienna, 1999; pp. 489-494.

29. O'Neill, M.P.; Bransby, M.F.; Randolph, M.F. Drag anchor fluke-soil interaction in clay. Can. Geotech. J. 2003, 40, 78-94. [CrossRef]

30. Elkhatib, S.; Randolph, M.F. The effect of interface friction on the performance of drag-in plate anchors. In Proceedings of the 5th International Symposium on Frontiers in Offshore Geotechnics, Perth, Australia, 2005; pp. 171-177.

31. Murff, J.D.; Randolph, M.F.; Elkhatib, S.; Kolk, H.J.; Ruinen, R.; Strom, P.J.; Thorne, C. Vertically loaded plate anchors for deepwater applications. In Proceedings of the 5th Int. Symp. on Frontiers in Offshore Geotechnics, Perth, Australia, 2005; pp. 31-48.

32. Aubeny, C.P.; Chi, C.-M. Mechanics of drag embedment anchors in a soft seabed. J. Geotech. Geoenviron. Eng. 2010, 136, 57-68. [CrossRef]

33. Gourvenec, S.M.; Vulpe, C.; Murthy, T.G. A method for predicting the consolidated undrained bearing capacity of shallow foundations. Géotechnique 2014, 64, 215-225. [CrossRef]

34. Fu, D.; Gaudin, C.; Tian, C.; Bienen, B.; Cassidy, M.J. Effects of preloading with consolidation on undrained bearing capacity of skirted circular footings. Géotechnique 2015, 65, 231-246. [CrossRef]

35. Corti, R.; Diambra, A.; Muir Wood, D.; Escribano Leiva, D.; Nash, D. Memory surface hardening model for granular soils under repeated loading conditions. J. Eng. Mech. 2016, 142. [CrossRef]

36. Wood, D.M. Soil Behaviour and Critical State Soil Mechanics; Cambridge University Press: Cambridge, UK, 1990.

37. Been, K.; Jefferies, M.G. A state parameter for sands. Géotechnique 1985, 35, 99-112. [CrossRef]

38. Gajo, A.; Muir Wood, D. Severn-Trent sand: A kineatic-hardening constitutive model: The q-p formulation. Géotechnique 1999, 49, 595-614. [CrossRef]

39. Manzari, M.T.; Dafalias, Y.F. A critical state two-surface plasticity model for sands. Géotechnique 1997, 47, 255-272. [CrossRef]

40. Rowe, R.K.; Davis, H. The behaviour of plate anchors in clay. Géotechnique 1982, 32, 9-23. [CrossRef]

41. Chow, S.H.; O'Loughlin, C.D.; Zhou, Z.; White, D.J.; Randolph, M.F. Penetrometer testing in a calcareous silt to explore changes in soil strength. Géotechnique 2020, 70, 1160-1173. [CrossRef]

42. Elkhatib, S. The Behaviour of Drag-in Plate Anchors in Soft Cohesive Soils. Ph.D. Thesis, The University of Western Australia, Perth, Australia, 2006. 